\title{
The pathophysiological basis of vascular disease
}

\author{
Alicia N. Lyle ${ }^{1} \cdot$ W. Robert Taylor ${ }^{1,2,3}$
}

Received: 8 January 2019 / Accepted: 8 January 2019 / Published online: 12 February 2019

(c) United States \& Canadian Academy of Pathology 2019

\section{The dynamic nature of the vascular wall}

Cardiovascular disease (CVD) remains the leading global cause of death and in 2017 an estimated 17.8 million deaths globally were attributed to CVD, and the number continues to grow [1]. Vascular diseases are the dominant component of CVD and adaptive and maladaptive remodeling of the vascular wall represent key processes in vascular diseases and are the major focus of therapeutic interventions. Vascular remodeling is an active process that leads to structural changes and requires cell growth, cell death, and cell migration, as well as degradation and reorganization of the extracellular matrix scaffold (ECM) in the vessel wall [2]. This adaptive process occurs in physiological conditions to maintain blood pressure hemostasis; however, in pathological conditions, such as hypertension, restenosis, and atherosclerosis, these adaptive changes instead initiate pathological vascular alterations [3, 4]. The cellular processes that contribute to the vascular remodeling that occurs in atherosclerosis and other CVD pathologies can be induced by inflammatory cytokines, hemodynamic stimuli, growth factors, and vasoactive hormones.

There are multiple cell types within the vessel wall that play a role in the vascular remodeling process: (1) endothelial cells (ECs), which comprise the innermost cell monolayer of the vessel and are in direct contact with blood flow, (2) vascular smooth muscle cells (VSMCs), which form the tunica media, provide structure, and regulate arterial

$\bowtie$ W. Robert Taylor

w.robert.taylor@emory.edu

1 Division of Cardiology, Department of Medicine, Emory University School of Medicine, Atlanta, GA, USA

2 Division of Cardiology, Atlanta Veterans Affairs Medical Center, Decatur, GA, USA

3 Wallace H. Coulter Department of Biomedical Engineering, Emory University and Georgia Institute of Technology, Atlanta, GA, USA vascular tone, and (3) fibroblasts and inflammatory cells, such as macrophages, are found in the adventitia layer, which functions to provide structural support to the blood vessel and is paramount to the outside-in inflammatory response to vascular injury that contributes to vessel remodeling [5]. It is now well understood that all of these cell types and layers of the vascular wall play a role in the vascular growth and repair adaptive processes. Within this editorial, we discuss the major contributions of each of these cell types on the vessel remodeling process and will emphasize recent progress in the field, highlighting the new findings published within this special issue on vascular biology.

\section{Endothelial cells}

The endothelial cell monolayer makes up the innermost cell layer of the artery and these arterial ECs are exposed to cyclical, perpetual wall shear stress as the direct result of blood flow across the ECs during the normal cardiac cycle. ECs are able to sense changes in shear stress through specialized mechanosensors that translate flow patterns into intracellular signals and this has been reviewed previously $[6,7]$. Atherosclerosis is a primary underlying cause of CVD and is a lipid and chronic inflammation driven disease [8], and it is now well accepted that local hemodynamic forces regulate site-specific atherosclerotic plaque formation. Atherosclerotic plaques primarily form at arterial sites exposed to disturbed or oscillatory shear stress, such as the outer walls of blood vessel bifurcations, the inner curvatures of vessels, and downstream of stenoses [9].

ECs in the regions of oscillatory flow have increased expression and activation of transcription factors that regulate proliferation, inflammatory protein expression, and reactive oxygen species (ROS) production, including nuclear factor- $\mathrm{\kappa B}(\mathrm{NF}-\mathrm{\kappa B})$, and activator protein (AP-1) [10]. The recent paper by Wang $Y$, et al. identifies a shearsensitive transcription factor, ZBTB46, expressed in ECs exposed to laminar shear stress that is downregulated in 
ECs in response to the disturbed flow in vitro and in vivo in the partial carotid ligation model of accelerated atherosclerosis [11]. Interestingly, they also show that ZBTB46 functions to block EC proliferation by suppressing cell cycle promoters like the cyclins (CCN A2, B1, D1, and E2), cyclin-dependent kinases (CDKs 1, 2, 4, and 6), and phospho-retinoblastoma (pRb), while also promoting increased expression of the cell cycle inhibitor p21 [11]. Altogether, these data suggest that ZBTB46 may be a key regulator of shear-mediated EC proliferation and atherosclerotic plaque formation [11].

High blood pressure, or hypertension, fundamentally occurs when the heart must pump against higher peripheral resistance, which occurs due to structural and functional changes in large conductive and small resistance arteries. Clinical cases of hypertension often present with enhanced sympathetic nerve activation [12], resulting in increased vasoconstriction and reduced vasodilation. There are a number of vasoactive molecules that also play a role in the maintenance of a balance between vasoconstriction and vasorelaxation. Nitric oxide $\left(\mathrm{NO}^{\circ}\right)$ is one of these molecules and is released by ECs to promote vasodilation via signaling to VSMCs. Laminar shear stress promotes $\mathrm{NO}^{\bullet}$ production through effects on both endothelial $\mathrm{NO}^{\bullet}$ synthase (eNOS) expression and activity through Krüppel-like factor 2 (Klf-

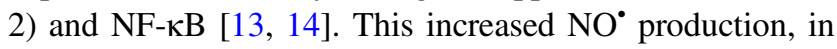
turn, inhibits further flow-induced NF-кB activation [14]. It is well known that chronic consumption of high-fat meals contributes to obesity, atherosclerosis, and endothelial dysfunction. Interestingly, Benson et al. demonstrate in their manuscript that a single high-fat meal in humans is enough to increase circulating triglyceride, cholesterol, free fatty acid levels, and circulating myeloperoxidase (MPO) activity and that these factors altogether contribute to impaired endothelial-dependent flow-mediated dilation [15]. It is known that MPO is able to reduce the bioavailability of $\mathrm{NO}^{\circ}$, thus inhibiting the vasodilatory functions of $\mathrm{NO}^{\circ}$. Interestingly, these new findings show that RBCs are one source of MPO and that free fatty acids induce MPO release from cells [15], which may ultimately contribute to endothelial-dysfunction and atherosclerosis.

\section{Vascular smooth muscle cells in vessel remodeling}

A primary vasoactive molecule that functions to regulate blood pressure through promotion of vasoconstriction is angiotensin II (Ang II). Numerous drugs developed to treat hypertension specifically target the renin-angiotensinaldosterone system (RAAS), including angiotensinconverting enzyme (ACE) inhibitors designed to block Ang II production. Clinical studies have shown that ACE inhibitors are beneficial in reversing conductive vascular remodeling caused by hypertension and decreasing arterial stiffness [16, 17]. In a seminal paper published in 1994 by Griendling et al. it was shown that Ang II stimulation of VSMCs increases NADPH oxidase (Nox) activity and reactive oxygen species (ROS) production [18], more specifically production of superoxide, which can be spontaneously or catalytically converted to the more stable and freely diffusible hydrogen peroxide [19]. This finding opened up an entire field of exploration into the role of reactive oxygen species and vascular health and disease [20, 21]. It has since been demonstrated that virtually all cells in the vessel wall produce ROS in varying amounts and in response to various stimuli [22, 23]. ROS are essential to the normal function of the vessel wall ( $\mathrm{NO}^{\bullet}$ for example), but a number of diseases are the result of an imbalance between the generation and scavenging of ROS, resulting in ROS levels in excess of endogenous antioxidant systems that contribute to a number of vascular pathologies [24]. Indeed, we now understand that superoxide generated by ECs, VSMCs, and macrophages can react with $\mathrm{NO}^{\circ}$ to generate the free radical peroxynitrite $\left(\mathrm{ONOO}^{\circ}-\right)$, which functionally decreases the bioavailability of $\mathrm{NO}^{\circ}$ and thus impairs vascular relaxation and vasodilation to promote vascular pathophysiology [25]. In pathologies such as hypertension, ROS also function as mediators of VSMC growth, which contributes to vascular remodeling.

The progression of hypertension involves two different types of vascular remodeling: inward eutrophic remodeling and hypertrophic remodeling [26]. Both forms of remodeling frequently coexist in different vascular beds. Eutrophic remodeling is characterized by a reduction in the vessel and thickening of the media, thus leading to increased media:lumen ratio, but usually shows little change in medial cross-sectional area. Hypertrophic remodeling is characterized by an increase in wall cross-sectional area and involves both an increase in VSMC size and enhanced accumulation of ECM proteins, such as collagen and fibronectin [27, 28]. The reorganization of the media that occurs in both types of remodeling (eutrophic and hypertrophic) is mediated by phenotype modulation of VSMCs, migration, cellular growth, apoptosis, and ECM production and rearrangement [25]. One of the consequences of hypertension is the induction of the expression of inflammation, fibrosis, and hypertrophy related genes in VSMCs.

VSMC remodeling in conductive and resistance arteries has recently been reviewed in detail [2], but we briefly discuss some of the contributing factors below. $\mathrm{I} \kappa \mathrm{B} \alpha$ (inhibitor of kappa B) functions to inhibit NF- $\mathrm{B}$ activation. Inhibitor kappa B kinase 2, or IKK2, functions by phosphorylating $\mathrm{I} \kappa \mathrm{B} \alpha$, which subsequently leads to I $\mathrm{K} \mathrm{B} \alpha$ ubiquitination and proteosomal degradation, allowing for NF- $\mathrm{BB}$ activation [29]. An interesting paradigm shift was recently proposed, 
which is that IKK2 can also serve as a myosin light chain kinase (MLCK) to promote phosphorylation of myosin light chain and initiation of VSMC contraction [30, 31]. However, it was unclear if IKK2 actually served as a MLCK or functioned to regulate myosin light chain phosphatase (MLCP) activity. Kwon et al. utilized rat mesenteric arteries and pressure myograph studies to demonstrate that IKK2 promotes vessel contractility by phosphorylating the myosin phosphatase target subunit MYPT1 and thus activating MLCP, suggesting that IKK2 plays a role in VSMC contractility that may be independent of NF-кB regulation [32].

Ang II-induced hypertension increases activity of the NF-кB, AP-1, and CRE-binding protein (CREB) transcription factors in VSMCs leading to the upregulation of monocyte chemoattractant protein 1 (MCP-1), transforming growth factor $\beta$ (TGF $\beta$ ), osteopontin (OPN), fibronectin (FN1), and collagens (COL). The actin cytoskeleton participates in cellular responses mediated by Ang II and mechanotransduction [33, 34]. More specifically, the actinbinding protein cofilin and its upstream activator slingshot phosphatase (SSH1) serve as key regulators of actin dynamics [35, 36]. SSH1 expression is upregulated during neo-intimal formation and has been linked to inflammatory signaling. The manuscript by Williams et al. utilized a novel $\mathrm{SSH}^{-1-}$ mouse to determine its importance in Ang II mediated vascular remodeling [37]. $\mathrm{SSH}^{-/-}$animal characterization showed no baseline differences in aortic contractility, mean blood pressure, or aortic structure [37]. Interestingly, the loss of SSH1 exacerbated baseline and AngII-induced increases in fibronectin deposition and osteopontin expression, as well as TGF $\beta 1$-induced expression of OPN. However, no differences in Ang II-mediated TGF $\beta$ or collagen production were detected between $\mathrm{SSH}^{-/-}$and control animals [37], suggesting that the actin regulator SSH1 functions to block increases in expression of specific fibrotic genes typically induced by Ang II in hypertension.

During remodeling, VSMCs undergo a process known as de-differentiation prior to migration and proliferation. One setting in which this commonly occurs is in neo-intimal hyperplasia. The de-differentiation of VSMCs from a contractile phenotype to a more proliferative and/or migratory phenotype typically precedes neo-intimal formation. Of note, ROS play a clear role in the regulation of VSMC phenotypic switching, which was comprehensively reviewed by Durgin et al. in "Redox control of vascular smooth muscle cell function and plasticity", in which the authors discuss the current understanding of the role of vascular Noxes in VSMC differentiation state [38]. In a study by Datla et al., they investigated the role of Poldip2, a multi-function protein with numerous roles in the vasculature, in the neo-intimal formation process [39]. They first establish in stage II human atherosclerotic plaques that
Polidp2 is highly expressed in the endothelium and in the underlying VSMCs, where Poldip2 co-localized with proliferating cell nuclear antigen (PCNA) [39]. Furthermore, they clearly show in a femoral-artery wire injury model that the lack of Poldip2 (heterozygous mice) decreases neointimal formation and ROS production in vivo and VSMC proliferation in vitro and in vivo, potentially via increased expression of the cell cycle inhibitor p21 [39]. One proposed mechanism by Datla et al. is the regulation of hydrogen peroxide production from Nox4 by Poldip2, since it was previously shown that Poldip2 increases Nox4 activity and ROS generation [40] and VSMC migration [41]. However, it is likely that other mechanisms are a play, since Poldip2 has also been linked to other functions [42]. Increased VSMC proliferation and migration are also required for arterial remodeling in the pulmonary setting. Dipeptidyl peptidase IV (DPP-4), also known as CD26, is a $110 \mathrm{kDa}$ type II transmembrane glycoprotein expressed by multiple cell types including VSMCs and ECs and DPP-4 inhibitors have been shown to have a plurality of beneficial effects, including suppression of VSMC proliferation and neo-intimal formation [43, 44]. $\mathrm{Xu}$ et al. investigated in their recent manuscript if DPP-4 inhibition would also have beneficial effects with respect to pulmonary artery remodeling [45]. Using an experimental model of pulmonary hypertension, they show that inhibition of DPP-4 with Sitagliptin attenuates right ventricular systolic pressure, right ventricle remodeling, hypertrophy of the medial layer in pulmonary arteries, and decreased inflammatory cell infiltration in vivo [45]. Additionally, they show that DPP4 inhibition blocks PDGF-mediated proliferation and migration in pulmonary VSMCs [45].

Excessive ROS production is also a primary contributor to diabetic vascular complications, including nephropathy and atherosclerosis, in part through chronic activation of the Janus kinase/Signal transducer and activator of transcription (JAK/STAT) signaling pathway. Unresolved activation of the JAK/STAT pathway increases expression of genes involved in cell proliferation, fibrosis, inflammation, and oxidative stress. Suppressors of cytokine signaling, known as the SOCS family of proteins, are endogenous regulators of JAK/STAT and are potential points for therapeutic intervention. Lopez-Sanz et al. investigated if SOCS-1 targeted therapies have beneficial effects in an STZ-induced murine model of diabetes [46]. They found that the use of adenovirus to overexpress SOCS-1 or a peptidomimetic was able to significantly lower superoxide production levels, DNA oxidation, and Nox subunit expression in vivo and in vitro [46]. Additional studies showed that SOCS-1 blocked high-glucose-mediated Nox complex assembly and Nox subunit expression [46]. SOCS-1 treatment also increased antioxidant enzyme expression, which correlated with a reduction in renal damage, atherosclerosis, and 
inflammation in vivo, suggesting that SOCS-1-targeted therapies may be an alternative strategy to limit oxidative stress and the progression of diabetic micro- and macrovascular complications [46].

Increased intrahepatic vascular resistance is thought to contribute to the progression of non-alcoholic fatty liver disease or steatosis and portal hypertension seems to precede increases in inflammation and fibrosis in this pathology. However, the exact mechanisms underlying vascular dysfunction in non-alcoholic fatty liver disease remain unknown. In the study from Van der Graaff et al. we learn that mean arterial blood pressure is lower while portal blood pressure is higher in a rat model of steatosis, and that maximal contraction force in aortic rings from these animals is also markedly reduced [47]. The authors ultimately demonstrate that, in contrast to liver cirrhosis, arterial hyporeactivity in steatosis is $\mathrm{NO}^{\circ}$-independent and, instead, appears to be mediated by cyclooxygenase 2 (COX-2)related mechanisms, whereas the increased intrahepatic vascular resistance appears to be related to hyper-reactivity to vasoconstrictive mediators [47]. However, it what vasoconstrictive mediators are at play in this setting remain to be established.

In addition to ROS, Notch signaling has been implicated in neo-intimal formation after vascular injury and Notch signaling is implicated in pro-inflammatory gene expression and is linked to atherosclerotic disease progression. However, Notch signaling in VSMCs during atherosclerosis development is not well established. Therefore, DavisKnowlton et al. set out to characterize VSMCs from human atherosclerotic lesions, to examine Notch expression levels, and to quantify cellular responses to Notch signaling [48]. Specifically, they found that medial and intimal VSMCs and plaque-derived VSMCs all express Notch2 and Notch3, whereas ECs primarily express Notch1 [48]. The Liaw group previously established that Notch signaling stimulated by Jagged 1 in healthy VSMCs induces differentiation/ contractile marker expression via both Notch2 and Notch3 and that Notch2 signaling suppressed VSMC proliferation [49]. In this recent study, they demonstrate that while Jagged1 stimulation of healthy VSMCs increased levels of smooth muscle $\alpha$-actin, this effect is lost in endartectomyderived VSMCs [48], the reasons for which remain to be investigated.

While many of the remodeling effects described above have been in the pathophysiologic setting, many of these same remodeling effects are also necessary for physiologic processes. The formation of collateral blood vessels is one example in which vascular remodeling is a good thing. Vessel occlusions caused by atherosclerosis, such as those that occur in peripheral artery disease, result in a loss of oxygen to the downstream tissues, also known as ischemia. The body's endogenous response to ischemia is to develop collateral blood vessels to bypass these sites of obstruction, restore blood flow and preserve tissue function [50]. Importantly, a patient's ability to form functional collateral vessels improves outcomes and survival [51]. Collateral vessel formation requires many of the same remodeling mechanisms described above working in concert [52-54], including cytokine signaling, ECM remodeling, and cell proliferation, and migration $[55,56]$. Often patients with other co-morbidities, such as diabetes, do not effectively form collateral blood vessels [57]. Therefore, understanding the biologic mechanisms that promote functional collateral vessel formation to restore blood flow may help identify novel therapeutic targets for non-invasive interventions. OPN, a secreted glyco-phospho-protein, was previously shown to be highly upregulated in response to ischemia in a ROS-dependent manner and was established as a critical mediator of post-ischemic collateral formation [58]. However, it was recently established that humans express three OPN isoforms as a result of alternative mRNA splicing to generate: OPNa, OPNb, and OPNc [59-61] and how each of these isoforms affect collateral formation is investigated in the manuscript by Lee et al. [62]. Utilizing a loss-of-function, gain-of-function in vivo approach, they demonstrate that OPN isoforms differentially affect limb perfusion and functional limb use, where OPNa and OPNc significantly improve both of these endpoints [62]. Furthermore, they go on to show that the OPNa- and OPNc-mediated increases in perfusion are primarily driven by increases in arteriogenesis via pro-migration and pro-survival effects on macrophages [62].

Vascular calcification contributes to decreases in vessel wall elasticity and is a highly regulated biological process involving the downregulation of VSMC contractile proteins, including SMA and SM22, and the upregulation of bone-related proteins like Runx2 and BMP2 [63]. Hyaluronan (HA) is an ECM structural protein that has been shown inhibit osteoblast differentiation. Kong et al. investigated if HA affects osteogenic differentiation and calcification of VSMCs and found that low and high molecular weight HA treatment significantly reduced VSMC calcification in a dose-dependent manner [64]. HA treatment decreased alkaline phosphatase activity, decreased expression of Runx2 and BMP2, and blocked BMP2 signaling, while overexpression of the HA synthase, HAS2, also inhibited calcification [64]. In contrast, inhibition of HA synthesis or knockdown of HAS2 increased calcification [64].

\section{Conclusion}

This special issue of Laboratory Investigation includes a spectrum of papers that address some of the fundamental 
issues in cardiovascular biology. One common theme for many of the manuscripts is the contribution of inflammation and repair to cardiovascular pathology. Indeed, many aspects of cardiovascular pathology reflect a maladaptive inflammatory process that is critical in the development and repair of the cardiovascular system. Evolution has selected for highly efficient developmental and repair mechanisms involving production of reactive oxygen species, calcification, recruitment of inflammatory cells, etc. that lead to adverse outcomes in the disease state. As a result, a better understanding of these fundamental mechanisms provides us with a broader understanding of the cardiovascular system throughout the life cycle.

\section{Compliance with ethical standards}

Conflict of interest The authors declare that they have no conflict of interest.

Publisher's note: Springer Nature remains neutral with regard to jurisdictional claims in published maps and institutional affiliations.

\section{References}

1. Global Burden of Disease Study (GBoDS). 2015 Results. Institute for Health Metrics and Evaluation (IHME). Seattle: University of Washington; 2016.

2. Brown IAM, Diederich L, Good ME, DeLalio LJ, Murphy SA, Cortese-Krott MM, et al. Vascular smooth muscle remodeling in conductive and resistance arteries in hypertension. Arterioscler Thromb Vasc Biol. 2018;38:1969-85.

3. Schiffrin EL. Vascular remodeling in hypertension: mechanisms and treatment. Hypertension. 2012;59:367-74.

4. Gibbons GH, Dzau VJ. The emerging concept of vascular remodeling. N Engl J Med. 1994;330:1431-8.

5. Maiellaro K, Taylor WR. The role of the adventitia in vascular inflammation. Cardiovasc Res. 2007;75:640-8.

6. Hahn C, Schwartz MA. Mechanotransduction in vascular physiology and atherogenesis. Nat Rev Mol Cell Biol. 2009;10: 53-62.

7. Baeyens N, Schwartz MA. Biomechanics of vascular mechanosensation and remodeling. Mol Biol Cell. 2016;27:7-11.

8. Libby P, Hansson GK. Inflammation and immunity in diseases of the arterial tree: players and layers. Circ Res. 2015;116:307-11.

9. Dhawan SS, Avati Nanjundappa RP, Branch JR, Taylor WR, Quyyumi AA, Jo H, et al. Shear stress and plaque development. Expert Rev Cardiovasc Ther. 2010;8:545-56.

10. Yurdagul A Jr, Finney AC, Woolard MD, Orr AW. The arterial microenvironment: the where and why of atherosclerosis. Biochem J. 2016;473:1281-95.

11. Wang Y, Sun HY, Kumar S, Puerta MDM, Jo H, Rezvan A. ZBTB46 is a shear-sensitive transcription factor inhibiting endothelial cell proliferation via gene expression regulation of cell cycle proteins. Lab Invest. 2018. https://doi.org/10.1038/s41374018-0060-5.

12. Grassi G, Mark A, Esler M. The sympathetic nervous system alterations in human hypertension. Circ Res. 2015;116:976-90.

13. Davis ME, Grumbach IM, Fukai T, Cutchins A, Harrison DG. Shear stress regulates endothelial nitric-oxide synthase promoter activity through nuclear factor kappaB binding. $\mathrm{J}$ Biol Chem. 2004;279:163-8.

14. Grumbach IM, Chen W, Mertens SA, Harrison DG. A negative feedback mechanism involving nitric oxide and nuclear factor kappa-B modulates endothelial nitric oxide synthase transcription. J Mol Cell Cardiol. 2005;39:595-603.

15. Benson TW, Weintraub NL, Kim HW, Seigler N, Kumar S, Pye J, et al. A single high-fat meal provokes pathological erythrocyte remodeling and increases myeloperoxidase levels: implications for acute coronary syndrome. Lab Invest. 2018;98:1300-10.

16. Girerd X, Giannattasio C, Moulin C, Safar M, Mancia G, Laurent S. Regression of radial artery wall hypertrophy and improvement of carotid artery compliance after long-term antihypertensive treatment in elderly patients. J Am Coll Cardiol. 1998;31: 1064-73.

17. Mahmud A, Feely J. Reduction in arterial stiffness with angiotensin II antagonist is comparable with and additive to ACE inhibition. Am J Hypertens. 2002;15:321-5.

18. Griendling KK, Minieri CA, Ollerenshaw JD, Alexander RW. Angiotensin II stimulates NADH and NADPH oxidase activity in cultured vascular smooth muscle cells. Circ Res. 1994;74:1141-8.

19. Fridovich I. Superoxide radical: an endogenous toxicant. Annu Rev Pharmacol Toxicol. 1983;23:239-57.

20. Garrido AM, Griendling KK. NADPH oxidases and angiotensin II receptor signaling. Mol Cell Endocrinol. 2009;302:148-58.

21. Lassegue B, Griendling KK. NADPH oxidases: functions and pathologies in the vasculature. Arterioscler Thromb Vasc Biol. 2010;30:653-61.

22. Lyle AN, Griendling KK. Modulation of vascular smooth muscle signaling by reactive oxygen species. Physiol (Bethesda). 2006;21:269-80.

23. Clempus RE, Griendling KK. Reactive oxygen species signaling in vascular smooth muscle cells. Cardiovasc Res. 2006;71: 216-25.

24. Forrester SJ, Kikuchi DS, Hernandes MS, Xu Q, Griendling KK. Reactive oxygen species in metabolic and inflammatory signaling. Circ Res. 2018;122:877-902.

25. Lee MY, Griendling KK. Redox signaling, vascular function, and hypertension. Antioxid Redox Signal. 2008;10:1045-59.

26. Schiffrin EL. Remodeling of resistance arteries in essential hypertension and effects of antihypertensive treatment. Am J Hypertens. 2004;17:1192-200.

27. Rizzoni D, Porteri E, Guefi D, Piccoli A, Castellano M, Pasini G, et al. Cellular hypertrophy in subcutaneous small arteries of patients with renovascular hypertension. Hypertension. 2000;35:931-5.

28. Duprez DA. Role of the renin-angiotensin-aldosterone system in vascular remodeling and inflammation: a clinical review. $\mathbf{J}$ Hypertens. 2006;24:983-91.

29. Schmid JA, Birbach A. IkappaB kinase beta (IKKbeta/IKK2/ IKBKB) - a key molecule in signaling to the transcription factor NF-kappaB. Cytokine Growth Factor Rev. 2008;19:157-65.

30. Ying Z, do Carmo JM, Xiang L, da Silva AA, Chen M, Ryan MJ, et al. Inhibitor kappaB kinase 2 is a myosin light chain kinase in vascular smooth muscle. Circ Res. 2013;113:562-70.

31. Chen M, Ma L, Hall JE, Liu X, Ying Z. Dual regulation of tumor necrosis factor-alpha on myosin light chain phosphorylation in vascular smooth muscle. Am J Physiol Heart Circ Physiol. 2015;308:H398-406.

32. Kwon Y, Choi SK, Byeon S, Lee YH. Involvement of inhibitor kappa B kinase 2 (IKK2) in the regulation of vascular tone. Lab Invest. 2018;98:1311-9.

33. Montenegro MF, Valdivia A, Smolensky A, Verma K, Taylor WR, San Martin A. Nox4-dependent activation of cofilin mediates VSMC reorientation in response to cyclic stretching. Free Radic Biol Med. 2015;85:288-94. 
34. Touyz RM, Yao G, Schiffrin EL. Role of the actin cytoskeleton in angiotensin II signaling in human vascular smooth muscle cells. Can J Physiol Pharmacol. 2005;83:91-7.

35. Nishita M, Tomizawa C, Yamamoto M, Horita Y, Ohashi K, Mizuno K. Spatial and temporal regulation of cofilin activity by LIM kinase and slingshot is critical for directional cell migration. $\mathbf{J}$ Cell Biol. 2005;171:349-59.

36. Huang TY, DerMardirossian C, Bokoch GM. Cofilin phosphatases and regulation of actin dynamics. Curr Opin Cell Biol. 2006;18:26-31.

37. Williams HC, Ma J, Weiss D, Lassegue B, Sutliff RL, San Martin A. The cofilin phosphatase slingshot homolog 1 restrains angiotensin II-induced vascular hypertrophy and fibrosis in vivo. Lab Invest. 2018. https://doi.org/10.1038/s41374-018-0116-6.

38. Durgin BG, Straub AC. Redox control of vascular smooth muscle cell function and plasticity. Lab Invest. 2018;98:1254-62.

39. Datla SR, L LH, Seidel-Rogol B, Dikalova AE, Harousseau M, Punkova L, et al. Poldip2 knockdown inhibits vascular smooth muscle proliferation and neointima formation by regulating the expression of PCNA and p21. Lab Invest. 2018. https://doi.org/10. 1038/s41374-018-0103-y.

40. Lyle AN, Deshpande NN, Taniyama Y, Seidel-Rogol B, Pounkova L, Du P, et al. Poldip2, a novel regulator of Nox4 and cytoskeletal integrity in vascular smooth muscle cells. Circ Res. 2009;105:249-59.

41. Datla SR, McGrail DJ, Vukelic S, Huff LP, Lyle AN, Pounkova $\mathrm{L}$, et al. Poldip2 controls vascular smooth muscle cell migration by regulating focal adhesion turnover and force polarization. Am J Physiol Heart Circ Physiol. 2014;307:H945-57.

42. Hernandes MS, Lassegue B, Griendling KK. Polymerase deltainteracting Protein 2: A Multifunctional Protein. J Cardiovasc Pharmacol. 2017;69:335-42.

43. Ervinna N, Mita T, Yasunari E, Azuma K, Tanaka R, Fujimura S, et al. Anagliptin, a DPP-4 inhibitor, suppresses proliferation of vascular smooth muscles and monocyte inflammatory reaction and attenuates atherosclerosis in male apo E-deficient mice. Endocrinology. 2013;154:1260-70.

44. Liu L, Liu J, Wong WT, Tian XY, Lau CW, Wang YX, et al. Dipeptidyl peptidase 4 inhibitor sitagliptin protects endothelial function in hypertension through a glucagon-like peptide 1dependent mechanism. Hypertension. 2012;60:833-41.

45. Xu J, Wang J, He M, Han H, Xie W, Wang H, et al. Dipeptidyl peptidase IV (DPP-4) inhibition alleviates pulmonary arterial remodeling in experimental pulmonary hypertension. Lab Invest. 2018;98:1333-46.

46. Lopez-Sanz L, Bernal S, Recio C, Lazaro I, Oguiza A, Melgar A, et al. SOCS1-targeted therapy ameliorates renal and vascular oxidative stress in diabetes via STAT1 and PI3K inhibition. Lab Invest. 2018;98:1276-90.

47. Van der Graaff D, Kwanten WJ, Couturier FJ, Govaerts JS, Verlinden W, Brosius I, et al. Severe steatosis induces portal hypertension by systemic arterial hyporeactivity and hepatic vasoconstrictor hyperreactivity in rats. Lab Invest. 2018;98: 1263-75.
48. Davis-Knowlton J, Turner JE, Turner A, Damian-Loring S, Hagler $\mathrm{N}$, Henderson $\mathrm{T}$, et al. Characterization of smooth muscle cells from human atherosclerotic lesions and their responses to Notch signaling. Lab Invest. 2018. https://doi.org/10.1038/s41374-0180072-1.

49. Boucher JM, Harrington A, Rostama B, Lindner V, Liaw L. A receptor-specific function for Notch2 in mediating vascular smooth muscle cell growth arrest through cyclin-dependent kinase inhibitor 1B. Circ Res. 2013;113:975-85.

50. Sabia PJ, Powers ER, Jayaweera AR, Ragosta M, Kaul S. Functional significance of collateral blood flow in patients with recent acute myocardial infarction. A study using myocardial contrast echocardiography. Circulation. 1992;85:2080-9.

51. Meier P, Gloekler S, Zbinden R, Beckh S, de Marchi SF, Zbinden S, et al. Beneficial effect of recruitable collaterals: a 10-year follow-up study in patients with stable coronary artery disease undergoing quantitative collateral measurements. Circulation. 2007;116:975-83.

52. Cai W, Schaper W. Mechanisms of arteriogenesis. Acta Biochim Biophys Sin (Shanghai). 2008;40:681-92.

53. Carmeliet P. Mechanisms of angiogenesis and arteriogenesis. Nat Med. 2000;6:389-95.

54. Fischer C, Schneider M and Carmeliet P. Principles and therapeutic implications of angiogenesis, vasculogenesis and arteriogenesis. Handb Exp Pharmacol. 2006;176:157-212.

55. Semenza GL. Vasculogenesis, angiogenesis, and arteriogenesis: mechanisms of blood vessel formation and remodeling. J Cell Biochem. 2007;102:840-7.

56. Troidl K, Schaper W. Arteriogenesis versus angiogenesis in peripheral artery disease. Diabetes Metab Res Rev. 2012;28(Suppl 1): 27-9.

57. Standards of medical care in diabetes-2015: summary of revisions. Diabetes Care. 2015;38 Supp 1:S4-S4.

58. Lyle AN, Joseph G, Fan AE, Weiss D, Landazuri N, Taylor WR. Reactive oxygen species regulate osteopontin expression in a murine model of postischemic neovascularization. Arterioscler Thromb Vasc Biol. 2012;32:1383-91.

59. Blasberg JD, Goparaju CM, Pass HI, Donington JS. Lung cancer osteopontin isoforms exhibit angiogenic functional heterogeneity. J Thorac Cardiovasc Surg. 2010;139:1587-93.

60. Coombes JD, Syn WK. Differential osteopontin functions: the role of osteopontin isoforms. Hepatology. 2015;62:323-4.

61. Goparaju CM, Pass HI, Blasberg JD, Hirsch N, Donington JS. Functional heterogeneity of osteopontin isoforms in non-small cell lung cancer. J Thorac Oncol. 2010;5:1516-23.

62. Lee GS, Salazar HF, Joseph G, Lok ZSY, Caroti CM, Weiss D, et al. Osteopontin isoforms differentially promote arteriogenesis in response to ischemia via macrophage accumulation and survival. Lab Invest. 2018. https://doi.org/10.1038/s41374-018-0094-8.

63. Lanzer P, Boehm M, Sorribas V, Thiriet M, Janzen J, Zeller T, et al. Medial vascular calcification revisited: review and perspectives. Eur Heart J. 2014;35:1515-25.

64. Kong Y, Liang Q, Chen Y, Yang P, Liu X, Li Y, et al. Hyaluronan negatively regulates vascular calcification involving BMP2 signaling. Lab Invest. 2018;98:1320-1332. 